\title{
Out-of-Pocket Household Expenditures on Medical Injections in Cambodia
}

\author{
Sachiko Ozawa ${ }^{1,2}$ (1) Tatenda T. Yemeke ${ }^{1} \cdot$ Alie F. Tawah $^{3,4} \cdot$ Vivek Kulkarni $^{5}$. \\ Manuela Villar Uribe ${ }^{6}$
}

Published online: 9 February 2018

(C) The Author(s) 2018. This article is an open access publication

\begin{abstract}
Background Cambodia has one of the highest rates of overall medical injection usage worldwide. Therapeutic injections, which are often unnecessary, contribute to the spread of blood-borne diseases.

Objective This study describes injection practices and associated household expenditures in rural northwest Cambodia.

Methods We assessed care-seeking patterns of surveyed adult family members who sought healthcare in the previous 30 days, including location of care, medical injection use, and out-of-pocket household expenditures for treatment. A regression model was used to explore the impact of injection use on out-of-pocket household expenditures. Results Among 480 households sampled, 298 included members who had been sick within the previous 30 days; a total of 342 episodes of care had been sought. Private
\end{abstract}

Sachiko Ozawa

ozawa@unc.edu

1 Division of Practice Advancement and Clinical Education, UNC Eshelman School of Pharmacy, University of North Carolina at Chapel Hill, CB\#7574, Beard Hall 115H, Chapel Hill, NC 27599, USA

2 Department of Maternal and Child Health, Gillings School of Global Public Health, University of North Carolina at Chapel Hill, Chapel Hill, NC, USA

3 Department of International Health, Johns Hopkins Bloomberg School of Public Health, Baltimore, MD, USA

4 Johns Hopkins Carey Business School, Baltimore, MD, USA

5 Division of Biology and Biological Engineering, California Institute of Technology, Pasadena, CA, USA

6 Health Nutrition and Population Global Practice, World Bank Group, Washington, DC, USA providers accounted for over $66 \%(n=226)$ of all episodes of care, with public and informal providers accounting for $20 \%(n=69)$ and $14 \%(n=47)$, respectively. Injections were administered in over $120(35 \%)$ episodes of care, with $81 \%$ of injections administered by private providers. Patients who received injections incurred total out-of-pocket household expenditures that were, on average, 126,590 Cambodian Riel (KHR) (US\$31.65) higher than those who did not receive injections $(p<0.01)$, equivalent to nearly half of the country's total annual health expenditure per capita. Receiving injections and perceived severity of illness were significantly associated with higher out-of-pocket household expenditures.

Conclusion This study found high levels of medical injection use, particularly among private healthcare providers, which was significantly associated with high healthcare expenditures. Reducing the number of medical injections would not only reduce disease transmission risk but also contribute to reduced healthcare costs and greater financial protection.

\section{Key Points for Decision Makers}

We observed high levels of medical injection use, particularly among private healthcare providers in Cambodia.

Injections increased average out-of-pocket expenditures per treatment significantly-the increase was equivalent to nearly half of the total annual health expenditure per capita.

Reducing the number of medical injections would lower the risk of disease transmission and prevent households from incurring large health expenditures. 


\section{Introduction}

The World Health Organization (WHO) estimates that up to $90 \%$ of global injection prescriptions are unnecessary and could be replaced by oral formulations [1, 2]. Oral formulations are often safer for patients and healthcare workers and more cost-effective than injectables [3, 4]. The overuse of medical injections is associated with unsafe injection practices and has been linked with transmission of blood-borne infectious diseases [5-7]. Globally, it is estimated that unsafe injections transmit 21 million hepatitis B infections, 2 million hepatitis $C$ infections and 260,000 HIV infections each year [8]. High use of injections is also associated with high rates of needle stick injuries among healthcare workers [4]. Safe and appropriate use of injections contributes to WHO's global patient safety challenge 'Medication Without Harm,' which aims to reduce severe avoidable medication-related harm by $50 \%$ globally over the next 5 years [9].

Cambodia has one of the highest rates of overall medical injection usage worldwide [10], where more than one in three individuals reported receiving a medical injection in the previous 12 months [11]. The overuse of injections and utilization of unsterile equipment has caused multiple outbreaks of HIV in rural Cambodian communities. For example, repeated use of HIV-infected syringes in Roka Commune in 2014 resulted in 242 confirmed new cases of HIV $[12,13]$. Moreover, $65 \%$ of injections are reported to be administered in patients' homes, which are not equipped for safe injections or to handle injection-related complications [11]. Overuse of injections is likely driven by a perception that injections have greater potency than oral formulations as well as providers' prescribing practices [14].

In Cambodia, healthcare expenditures are the second greatest household expense, and $74 \%$ of healthcare expenditures are paid out-of-pocket [15]. Health expenditures cause medical impoverishment among $4.1 \%$ of Cambodian families each month [16]. To reduce out-ofpocket health expenditures, Cambodia has introduced health equity funds (HEF), which provide free healthcare at public health facilities for the poorest individuals, covering about one-quarter of the population $[15,17,18]$. Community-based health insurance (CBHI) schemes have also been introduced, but they cover less than $1 \%$ of the population, while other private health insurance schemes cover an even smaller proportion of the population $[15,19,20]$. Moreover, neither HEF nor CBHI cover services provided by private healthcare providers, where more than twothirds of care is sought and where most injections are administered $[10,21]$. Hence, even with insurance and supply-side subsidies, patients are still at risk of incurring large injection-related health expenditures.
We conducted a household survey in northwest Cambodia to gain an understanding of the utilization of and financial burden from medical injections. We describe healthcare injection practices across public, private and informal providers, including frequency of injections and associated out-of-pocket health expenditures by households. Findings from the study can inform the design of interventions and policies to reduce the use of injections, reduce catastrophic health expenditures and highlight the financial burden that injections impose on Cambodian households.

\section{Methods}

We conducted a household survey in two rural health operational districts (ODs) in Cambodia: Thmar Pouk OD in Banteay Meanchey province and Samrong OD in Oddar Meanchey province. Eight villages in each OD were randomly selected based on the size of the village population, the health centre coverage (one village per health centre) and the distance between the village and the nearest health centre. We surveyed 480 households using a standard cluster sampling methodology, interviewing 30 households in each of the 16 villages. Participants were heads of households or their spouses aged $>18$ years who were not medical practitioners. Only households that reported having a sick member in the previous 30 days were included in this analysis.

Each healthcare visit was counted as an episode of care, and up to three healthcare visits were recorded per household. From each household, we collected basic demographic data about the respondent, as well as information about the family member's illness, healthcare utilization and self-reported total out-of-pocket household expenditures for each episode of care. The health providers/facilities where sick household members received care were categorized as either public, private or informal. Public facilities and/or providers included hospitals in Phnom Penh, provincial hospitals, Thmar Pouk district hospital or health centres. Private facilities and/or providers included private hospitals, private clinics, home/office of trained health workers, visits by trained health workers, and pharmacies. The remaining facilities and/or providers were classified as informal: shops/markets selling drugs, Kru Khmer (traditional healers), monks/religious leaders, traditional birth attendants or other informal providers.

Household expenditures included spending on provider and facility charges, medications, travel to seek healthcare, meals and other incidental costs. We asked about health insurance status, as this area is covered by a CBHI scheme operated by a local non-governmental organization called Cambodian Association for Assistance to Families 
and Widows (CAAFW). This voluntary insurance coverage is available for enrolled families and can be used only at public providers. Coverage under the scheme includes most services at health centres and referral hospitals in the area.

Data were analysed by episode of care using the Stata software (StataCorp. 2015 Stata Statistical Software: Release 14. College Station, TX, USA). We used Chi squared and $t$ tests to examine whether there were any differences between respondents' demographic characteristics and episode of care for those who received injections and those who did not. We summarized the total out-of-pocket household expenditures per episode of illness. Cambodian Riel (KHR) were converted to US dollars at KHR4000 per US\$1 [22]. Some respondents were unable to recall their households' expenditures, so we analyzed the subset with data on household expenditures. We compared household expenditures across four categories of treatment: no treatment, received oral medications only, received injections only and received both injections and oral medications.

We used a linear regression model to assess the impact of receiving injections on out-of-pocket household expenditure per episode of illness while controlling for respondent characteristics. Specifically, our analysis controlled for respondent age, health provider/facility, perceived severity of illness and receiving oral medications, which were statistically significantly different between respondents who received injections and those who did not.

\section{Results}

We recruited 480 households, 312 of which had members who had been sick in the 30 days preceding the survey; 298 households (95\%) had sought care in the previous 30 days. Among households that sought care, 342 episodes of care were received from health providers. Of the episodes of care, $226(66 \%)$ were at a private provider, $69(20 \%)$ at a public provider and $47(14 \%)$ at an informal provider. While most care was sought during one visit, 44 (13\%) were repeat visits for the same illness. Figure 1 summarizes the flow of households recruited and their care-seeking patterns.

Injections were administered in $35 \%(n=120)$ of all treatments sought. Household members were more likely to have received injections if they perceived their illness as severe $(55 \%, n=46 ; p<0.001)$ and if they received care at a private provider $(81 \%, n=98 ; p<0.001)$. While those who received injections received fewer oral medications $(p<0.001), 86 \%(n=103)$ received both injections and oral medications. Respondents in households that received injections were older than respondents in households that did not (43.5 vs. 40.1 years, respectively; $p=0.02$ ).

Table 1 summarizes the characteristics of respondents and compares those who received injections with those who did not, by episode of care. Socioeconomic status and household health insurance status were not significantly different between households that received injections and those that did not.

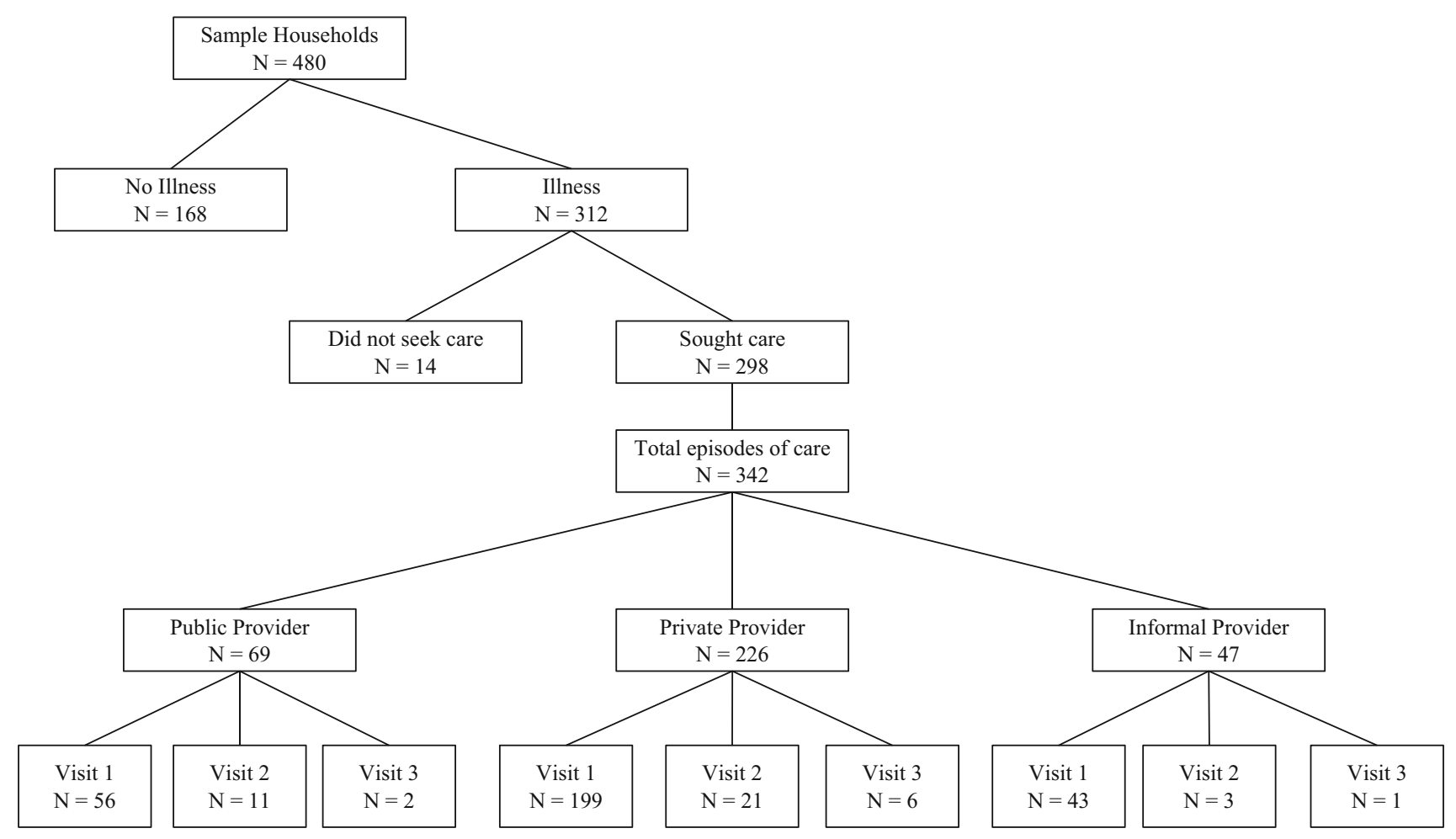

Fig. 1 Care-seeking patterns of surveyed households 
Table 1 Characteristics of survey respondents by injection use

\begin{tabular}{|c|c|c|c|}
\hline Respondent characteristics & Received injections $(n=120)$ & Did not receive injections $(n=222)$ & $p$ values \\
\hline Age, years & $43.54 \pm 13.44$ & $40.09 \pm 12.66$ & $0.019 *$ \\
\hline \multicolumn{4}{|l|}{ Sex } \\
\hline Male & 10.83 & 8.56 & \multirow[t]{2}{*}{0.491} \\
\hline Female & 89.17 & 91.44 & \\
\hline \multicolumn{4}{|l|}{ Marital status } \\
\hline Single & 2.50 & 2.25 & \multirow[t]{3}{*}{0.817} \\
\hline Married & 79.17 & 81.98 & \\
\hline Widowed/divorced/separated & 18.33 & 15.77 & \\
\hline Years of schooling & $2.75 \pm 3.05$ & $2.38 \pm 2.76$ & 0.255 \\
\hline \multicolumn{4}{|l|}{ Current health status } \\
\hline Excellent & 0.83 & 0.00 & \multirow[t]{5}{*}{0.430} \\
\hline Very good & 4.17 & 5.41 & \\
\hline Good & 23.33 & 19.37 & \\
\hline Fair & 53.33 & 51.35 & \\
\hline Poor & 18.33 & 23.87 & \\
\hline \multicolumn{4}{|l|}{ Perceived illness severity } \\
\hline Severe & 55.00 & 19.82 & \multirow[t]{2}{*}{$<0.001 *$} \\
\hline Not severe & 45.00 & 80.18 & \\
\hline Received oral medication & 86.67 & 98.20 & $<0.001 *$ \\
\hline \multicolumn{4}{|l|}{ Health facility/provider type } \\
\hline Public & 15.00 & 22.97 & \multirow[t]{3}{*}{$<0.001^{*}$} \\
\hline Private & 81.67 & 57.66 & \\
\hline Informal & 3.33 & 19.37 & \\
\hline Households with health insurance & 15.00 & 22.52 & 0.096 \\
\hline \multicolumn{4}{|l|}{ Socioeconomic status } \\
\hline Low & 15.83 & 23.87 & \multirow[t]{5}{*}{0.199} \\
\hline Low-medium & 17.50 & 22.97 & \\
\hline Medium & 21.67 & 16.67 & \\
\hline Medium-high & 23.33 & 18.47 & \\
\hline High & 21.67 & 18.02 & \\
\hline
\end{tabular}

Data are presented as $N$ or mean \pm standard deviation unless otherwise indicated

*Significant at $p<0.05$

Total out-of-pocket household expenditures per episode of care ranged widely by type of treatment received (Table 2). Households whose members received both oral medication and injections had the highest average out-ofpocket household expenditures (mean KHR208,726, US\$52.18). Households whose members only received injections had the second highest total out-of-pocket household expenditure (mean KHR102,666, US\$25.67). Those who received only oral medication incurred the lowest total out-of-pocket household expenditures (KHR34,938, US\$8.41). In only four episodes of care, neither oral medication nor injections were given.

Findings from our linear regression model examining the impact of receiving injections on total out-of-pocket household expenditure are summarized in Table 3. Receiving injections was significantly associated with
KHR126,590 (US\$31.65) more total out-of-pocket household expenditure per episode of care, controlling for household respondent's age, receiving oral medication, health provider type and severity of illness.

\section{Discussion}

Our study found that sick household members received injections as part of their treatment in more than one-third of all care sought. Private providers administered the majority $(81 \%)$ of these injections. Most patients $(86 \%)$ who received injections also received oral medications. This suggests that injections are not being administered as substitutes for oral formulations but are rather being added to the treatment mix. 
Table 2 Household out-of-pocket expenditures per episode of care by treatment

\begin{tabular}{lllll}
\hline & $\begin{array}{l}\text { Received injections } \\
\text { only }\end{array}$ & $\begin{array}{l}\text { Received injections and oral } \\
\text { medication }\end{array}$ & $\begin{array}{l}\text { Received oral medication } \\
\text { only }\end{array}$ & $\begin{array}{l}\text { Received neither injection or oral } \\
\text { medication }^{\text {b }}\end{array}$ \\
\hline$N$ & 15 & 101 & 215 & 4 \\
Mean \pm SD, KHR & $102,666 \pm 120,352$ & $208,726 \pm 344,625$ & $34,938 \pm 62,064$ & $167,500 \pm 173,853$ \\
Median, KHR & 75,000 & 100,000 & 12,500 & 125,000 \\
Mean \pm SD, US\$ & $25.67 \pm 30.09$ & $52.18 \pm 86.16$ & $8.73 \pm 15.52$ & $41.88 \pm 43.46$ \\
Median, US\$ & 18.75 & 25.00 & 3.13 & 31.25 \\
\hline
\end{tabular}

Reports on the subset of episodes of care with household expenditure data $(N=335)$

KHR Cambodian Riel, SD standard deviation

${ }^{\mathrm{a}}$ Conversion at KHR4000 = US $\$ 1$

${ }^{\mathrm{b}}$ No injections or oral medications were given in 4 episodes of care, where costs were incurred for other types of medical procedures

Table 3 Summary of generalized linear model of predictors of total visit costs

\begin{tabular}{lccc}
\hline Variable & Coefficient & Standard error & $p$ value \\
\hline Received injection(s) & $126,590.20$ & $24,873.59$ & $<0.001^{*}$ \\
Respondent's age & 358.45 & 838.91 & 0.669 \\
Health facility/provider type & -5433.27 & $18,713.05$ & 0.772 \\
Perceived illness severity & $95,373.28$ & $24,957.89$ & $<0.001^{*}$ \\
Received oral medication & $36,181.88$ & $48,107.24$ & 0.453 \\
Adjusted $R^{2}$ & 0.1545 & & 13.20 \\
$F$ & & & \\
\hline
\end{tabular}

*Significant at $p<0.05$
Receiving injections was significantly associated with higher total out-of-pocket household expenditure per episode of care, at an average of US\$31.65 (95\% confidence interval [CI] 19.41-43.88) more. In comparison, Cambodia's current total annual health expenditure per capita is US\$70, including government expenditures [23]. Cambodia's current out-of-pocket expenditure per capita is US\$41 per year [23]. These results are significant, as Cambodia seeks to improve the quality of healthcare and reduce healthcare-driven poverty. While this study cannot determine what proportion of the injections given were appropriate, the high prevalence of injections suggests that some are likely to be unnecessary and/or inappropriate. The higher costs incurred by patients for injections could potentially be prevented by reducing injection use and improving the standard of care.

The proportion of households in our study that sought care $(95 \%)$ and the types of health provider from which they sought the care (mostly private) was consistent with findings from the Cambodia demographic health survey (DHS) conducted in 2014 [11]. The prevalence of injection use $(35 \%)$ found in our study was higher than the 2014 DHS estimate for Oddar Meanchey province (24\%) and lower than the estimate for Banteay Meanchey province (39\%) [11]. The prevalence of injection use in the study was also similar to that found among patients using injections for malaria treatment in northeast Cambodia [24]. Some study design differences may explain the recorded prevalence. While the DHS survey recorded injections received within the previous 12 months among people aged 15-49 years, our study recorded injections received within the previous 30 days regardless of recipient's age. Furthermore, the DHS survey asked respondents directly about any injections received, whereas our study asked household representatives, who may not have received the injections themselves.

The highest average total out-of-pocket household expenditures in our study (KHR208,726, US\$50.26) were incurred for episodes of care in which patients received both injections and oral medications. In contrast, average total health treatment costs in the 2014 DHS survey were US $\$ 57.08$ in Banteay Meanchey and US\$55.33 in Oddar Meanchey. The healthcare expenditure estimates reported in the DHS survey were across all ages and thus can be directly compared to those in our sample.

High medical injection use in developing countries such as Cambodia is spurred by the perception that injections are a more potent and effective form of treatment than oral pills [25], leading to high demand from patients [24, 26-28]. High use of medical injections in Cambodia 
has been documented for malaria treatment, particularly in the private sector, with $32 \%$ of households surveyed receiving injections for their last malaria treatment [24]. Among those receiving injections, single-dosage injections were popular and sought from providers, likely contributing to increased drug resistance [24]. Perverse financial incentives are an important factor contributing to overuse of injections, with higher revenue from injections and other expensive treatments leading to irrational prescribing behaviours $[29,30]$. There is evidence that promotional practices and incentives from pharmaceutical companies also drive irrational drug use, including over-prescription of injections [31-33].

Reducing the overuse of injections in Cambodia will require a multisector approach, given the multi-faceted causes of injection overuse. Interventions to reduce the use of medical injections will likely need to target behaviour change among both medical providers and patients, similar to those proven successful and cost-effective in other settings [34-36]. Nevertheless, even with educational interventions, it is difficult to change the behaviour of all actors involved in injection use. Thus, at least in the short term, it may be imperative to adopt strategies that work to make current injections safer, while working to reduce injection use in the long term. The WHO, under the auspices of the Safe Injection Global Network (SIGN), has established the cost-effectiveness of this two-pronged intervention approach [37].

A number of study limitations must be noted. The study did not obtain clinical diagnoses of illness for which household members sought care, thus we were unable to definitively ascertain the appropriateness of injections received. Our estimates of out-of-pocket household expenditure were obtained through self-reporting and are thus subject to recall bias. Furthermore, patient household expenditures are only part of a broader range of health system costs associated with injection overuse. Costs borne by government through supply-side financing of injections, costs of infections caused by unnecessary injections and economic costs incurred by healthcare workers through needle stick injuries also need consideration. Future studies should consider a broader perspective of costs associated with injections and use administrative data and other health records, where available, to improve the accuracy of estimates. Finally, the regression assumed each episode of care was independent, but this assumption may not hold in instances where the same household member received care in multiple visits.

This study adds to the literature on the overuse of therapeutic injections in Cambodia. Furthermore, the study demonstrates the high economic burden, from the household perspective, associated with receiving injections. Together with evidence on public health harm, the findings on the economic burden on patients in this study highlight the need for interventions to reduce the use of injections in health facilities, especially among private providers. In addition to reducing the risk of injection-transmitted infections and injection-related sequelae, such interventions may have important cost-saving implications for patients and the health system overall, which is a key outcome in the push for universal health coverage in Cambodia [38].

Acknowledgements The authors are grateful to Ir Por and the team at the Cambodia National Institute of Public Health for guidance and data collection efforts.

Author Contributions SO conceptualized and planned the study. SO, VK and MVU contributed to the acquisition of data. SO, VK, AT and TY analysed and interpreted the study data. All authors contributed to the drafting and critical revision of the manuscript. All authors approved the final submitted version.

Data Availability Statement The datasets generated during and/or analysed in the current study is available in the study repository: https://github.com/ghepunc/cambodia-injections.

\section{Compliance with Ethical Standards}

Funding This study was performed with financial support from Johns Hopkins Center for Global Health (Building informed trust: injections and health insurance).

Ethical approval and consent The study was reviewed and approved by the National Ethics Committee for Health Research in Cambodia (reference number $148 \mathrm{NECHR}$ ) and the Institutional Review Board of Johns Hopkins Bloomberg School of Public Health and was conducted in accordance with the ethical standards of the Declaration of Helsinki. Informed consent was obtained from all respondents.

Conflict of interest SO, TY, AT, VK and MVU have no conflicts of interest.

Open Access This article is distributed under the terms of the Creative Commons Attribution-NonCommercial 4.0 International License (http://creativecommons.org/licenses/by-nc/4.0/), which permits any noncommercial use, distribution, and reproduction in any medium, provided you give appropriate credit to the original author(s) and the source, provide a link to the Creative Commons license, and indicate if changes were made.

\section{References}

1. World Health Organization. The world medicines situation. Geneva: World Health Organization; 2004 [cited 2018 February 1]. http://apps.who.int/medicinedocs/en/d/Js6160e/.

2. World Health Organization. Against all reason: misuse and overuse of injections Geneva: World Health Organization; 2017 [cited 2017 April 3]. http://www.who.int/injection_safety/about/ resources/Misuse/en/.

3. Cyriac JM, James E. Switch over from intravenous to oral therapy: a concise overview. J Pharmacol Pharmacother. 2014;5(2):83-7. 
4. Cooke CE, Stephens JM. Clinical, economic, and humanistic burden of needlestick injuries in healthcare workers. Med Device (Auckl NZ). 2017;10:225-35.

5. Pépin J, Abou Chakra CN, Pépin E, Nault V. Evolution of the global use of unsafe medical injections, 2000-2010. PLoS ONE. 2013;8(12):e80948.

6. Nsimba SED, Gesase AP, Massele AY. Dangers of injections overuse in developing countries with a high HIV/AIDS prevalence: a review on HIV risk hazards, traumatic effects and other blood borne infections. Asian Pac J Trop Dis. 2011;1(2):158-63.

7. Gore C, Lazarus JV, Peck RJJ, Sperle I, Safreed-Harmon K. Unnecessary injecting of medicines is still a major public health challenge globally. Trop Med Int Health TM IH. 2013;18(9):1157-9.

8. Hauri AM, Armstrong GL, Hutin YJ. The global burden of disease attributable to contaminated injections given in health care settings. Int J STD AIDS. 2004;15(1):7-16.

9. World Health Organization. Medication without harm-global patient safety challenge on medication safety. Geneva: World Health Organization; 2017 [cited 2018 February 1]. http://www. who.int/patientsafety/medication-safety/medication-without-harmbrochure/en/.

10. Vong S, Perz JF, Sok S, Som S, Goldstein S, Hutin Y, et al. Rapid assessment of injection practices in Cambodia, 2002. BMC Public Health. 2005;5:56.

11. National Institute of Statistics Cambodia, Directorate General for Health Cambodia, ICF International. Cambodia demographic and health survey 2014. Phnom Penh: National Institute of Statistics Cambodia, Directorate General for Health Cambodia, and ICF International; 2015.

12. Gokhale RH, Galang RR, Pitman JP, Brooks JT. A tale of 2 HIV outbreaks caused by unsafe injections in Cambodia and the United States, 2014-2015. Am J Infect Control. 2017;45(2):106-7.

13. Saphonn V, Fujita M, Samreth S, Chan S, Rouet F, Khol V, et al. Cluster of HIV infections associated with unsafe injection practices in a rural village in Cambodia. JAIDS J Acquir Immune Defic Syndr. 2017;75(3):e82-e86.

14. UNICEF. Health service access among poor communities in Phnom Penh. Phnom Penh: UNICEF; 2009.

15. Annear P, Grundy J, Ir P, Jacobs B, Men C, Nachtnebel M et al. The kingdom of Cambodia health system review. Health systems in transition, vol. 5, no. 2. Manila: Asia Pacific Observatory on Health Systems and Policies, World Health Organization; 2015.

16. Anuranga C, Chandrasiri J, Wickramasinghe R, Rannan-Eliya RP. The impact of out-of-pocket expenditures on families and barriers to use of maternal and child health services in Cambodia: evidence from the Cambodia socio-economic survey 2007. Manila: Asian Development Bank; 2007.

17. Ensor T, Chhun C, Kimsun T, McPake B, Edoka I. Impact of health financing policies in Cambodia: a 20 year experience. Soc Sci Med. 2017;177:118-26.

18. Bigdeli M, Jacobs B, Men CR, Nilsen K, Van Damme W, Dujardin B. Access to treatment for diabetes and hypertension in rural Cambodia: performance of existing social health protection schemes. PLoS ONE. 2016;11(1):e0146147.

19. Ozawa S, Walker DG. Trust in the context of community-based health insurance schemes in Cambodia: villagers' trust in health insurers. Adv Health Econ Health Serv Res. 2009;21:107-32.

20. Ozawa S, Grewal S, Bridges JF. Household size and the decision to purchase health insurance in cambodia: results of a discretechoice experiment with scale adjustment. Appl Health Econ Health Policy. 2016;14(2):195-204.
21. Ozawa S, Walker DG. Comparison of trust in public vs private health care providers in rural Cambodia. Health Policy Plan. 2011;26(Suppl 1):i20-9.

22. Cambodia; NBo. Exchange rate: National Bank of Cambodia; 2017 [cited 201712 October]. https://www.nbc.org.kh/english/ economic_research/exchange_rate.php.

23. Global Health Observatory data repository: health expenditure per capita, by country, 1995-2015 Cambodia [Internet]. World Health Organization, 2015 [cited February 1, 2018]. http://apps. who.int/nha/database/ViewData/Indicators/en.

24. Gryseels C, Uk S, Erhart A, Gerrets R, Sluydts V, Durnez L, et al. Injections, cocktails and diviners: therapeutic flexibility in the context of malaria elimination and drug resistance in northeast Cambodia. PLoS ONE. 2013;8(11):e80343.

25. Reeler AV. Anthropological perspectives on injections: a review. Bull World Health Organ. 2000;78(1):135-43.

26. Altaf A, Fatmi Z, Ajmal A, Hussain T, Qahir H, Agboatwalla M. Determinants of therapeutic injection overuse among communities in Sindh, Pakistan. J Ayub Med Coll Abbottabad JAMC. 2004;16(3):35-8.

27. Janjua NZ, Hutin YJ, Akhtar S, Ahmad K. Population beliefs about the efficacy of injections in Pakistan's Sindh province. Public Health. 2006;120(9):824-33.

28. Choi K-H, Park S-M, Lee J-H, Kwon S. Factors affecting the prescribing patterns of antibiotics and injections. J Korean Med Sci. 2012;27(2):120-7.

29. Li Y, Xu J, Wang F, Wang B, Liu L, Hou W, et al. Overprescribing in China, driven by financial incentives, results in very high use of antibiotics, injections, and corticosteroids. Health Aff (Proj Hope). 2012;31(5):1075-82.

30. Reynolds L, McKee M. Serve the people or close the sale? Profitdriven overuse of injections and infusions in China's marketbased healthcare system. Int $J$ Health Plann Manag. 2011;26(4):449-70.

31. Kshirsagar NA. Rational use of medicines: cost consideration \& way forward. Indian J Med Res. 2016;144(4):502-5.

32. Yuan $\mathrm{S}$. China should reduce the overuse of intravenous infusion. BMJ Br Med J. 2014;348:g1262.

33. Giri BR, Shankar PR. Learning how drug companies promote medicines in Nepal. PLoS Med. 2005;2(8):e256.

34. Bhunia R, Hutin Y, Ramkrishnan R, Ghosh PK, Dey S, Murhekar M. Reducing use of injections through interactional group discussions: a randomized controlled trial. Indian Pediatr. 2010;47(5):409-14.

35. Massele AY, Mashalla YJ, Kayombo EJ, Mwaiselage JD, Mwamba NE, Kaniki I. Reducing therapeutic injection overuse through patients-prescribers interaction group discussions in Kinondoni District, Dar es Salaam, Tanzania. Tanzan J Health Res. 2011;13(1):69-73.

36. Dziekan G, Chisholm D, Johns B, Rovira J, Hutin YJ. The costeffectiveness of policies for the safe and appropriate use of injection in healthcare settings. Bull World Health Organ. 2003;81(4):277-85.

37. World Health Organization. Safe injection global network Geneva, Switzerland: World Health Organization; 2017 [cited 2017 November 1]. http://www.who.int/medical_devices/ collaborations/network/en/.

38. Jordanwood T. Moving towards universal health coverage in Cambodia: University Research Co.; 2015 [cited 201729 September]. http://www.urc-chs.com/news/moving-towardsuniversal-health-coverage-cambodia. 